\title{
Morgagni Stewart Morel syndrome - Additional features
}

\author{
Mallikarjuna Nallegowda, U. Singh, Meeka Khanna, S. L. Yadav, Ashesh Ray Choudhary, \\ Alok Thakar*
}

Departments of Physical Medicine \& Rehabilitation and *Otorhinolaryngology, All India Institute of Medical Sciences, New Delhi -110029, India

\begin{abstract}
A case of Morgagni Stewart Morel syndrome with progressive depression in frontal bone, headache, transient monoparesis, obesity; imbalance, neuro psychiatric symptoms and recurrent disc prolapse with absent right radial pulse is discussed. This syndrome was first mentioned 235 years back, but till now exact pathology is not known. Balance assessment using dynamic posturography was done, which revealed abnormal vestibular function. To our knowledge this is the first case examined for Dynamic Posturography.
\end{abstract}

Key Words: Morgagni-Stewart-Morel syndrome, Balance, Dynamic Posturography, Headache, Backache, Rehabilitation, Radial Pulse

\section{Introduction}

Morgagni Stewart Morel (MSM) syndrome was diagnosed nearly 235 years ago. Morgagni and Santorini first described it in an obese female patient during autopsy who had hirsutism and thickening of inner table of a skull. In 1928 Stewart added neuropsychiatric problems. The first living case was reported by Morel in 1930. ${ }^{[1]}$ Due to its wide range of symptoms and endocrinal dysfunctions it was also called as metabolic craniopathy. There was a common association with diabetes mellitus, diabetes insipidus, and hyperparathyroidism. ${ }^{[1]}$ Because of its magnitude of problems many patients are usually misdiagnosed and patients have high degree of morbidity. Many specialists misdiagnosed the case presented here before she reported to us. This is the second case of its type reported from India. Apart from the diagnosis, dynamic posturography was done which showed vestibular dysfunction.

\section{Case Report}

A 37 years old obese staff nurse was referred from a northeast state hospital for her backache, from which she was suffering for the last 15 years. Pain was severe for the last 18 months when the patient reported in May 2001. She was admitted in a hospital in south India and diagnosed as vascular headache with prolapsed intervertebral disc of L4-L5 and managed conservatively. Later she had paraparesis in April 2000 and June 2001 with urinary retention. There was recovery with conservative management after 4-6 weeks but urinary symptoms persisted. The patient was also suffering from headache and transient monoparesis for the last 11 years. She also noticed progressive depression in the midline of forehead. Her symptoms worsened with onset of giddiness, unsteadiness of gait, nausea, vomiting and photophobia for the last five years. She used to go into deep sleep for about 6-24 hours after episodes of giddiness and vomiting. In addition, she had on and off pain in the breasts, which was relieved after expressing milk. She had weakness in all four limbs, more on the right side and she was constantly tired. In 2002 May she had two episodes of hematuria, and later ultrasound examination revealed renal stones. She was mentally disturbed and her body weight increased inspite of dieting and exercises. A previous CT spine showed disc prolapse of L4-L5.

On examination she was obese, depressed, BMI was 35. There was depression in the midline of the frontal bone (Figure 1). Right radial pulse was absent. Right upper limb and lower limb power was 3/5; right ankle reflex was depressed and there was sensory deficit in L4-

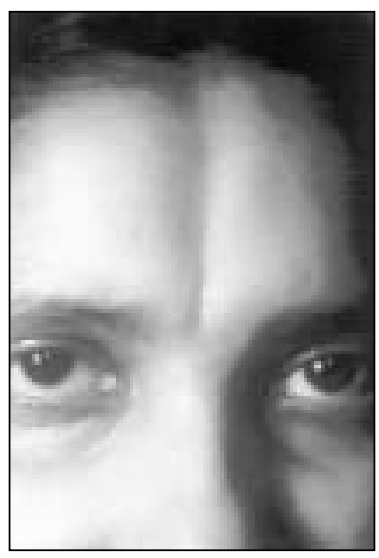

Figure 1: Depression in the midline of frontal bone 
S1 region. Straight leg raising test was positive with $30^{\circ}$ on right side and $40^{\circ}$ on left side.

Routine investigations were normal except raised ESR-50 mm/ $1^{\text {st }}$ hour. Serum cortisol, prolactin, progesterone, estradiol, T3, T4, TSH and GTT were within normal limits. The skeletal survey showed thickening of inner table of frontal bone with sclerosis suggestive of Hyperostosis frontalis interna, and spondylolisthesis of L5 over $\mathrm{S} 1$ with spondylolysis. MRI brain was normal. MRI of spine showed disc prolapse of L5-S1 and L4-L5 with mild thecal compression. Bone scan showed relative increase of radiotracer concentration in left frontal bone. The vestibulometry showed bilateral hypoactive response to caloric stimulation suggesting bilateral hypoactive labyrinth and audiometry revealed bilateral mild sensory hearing loss.

The balance assessment was done in the department of PMR using dynamic posturography. On unstable surface and absent visual feedback patients' equilibrium score was $12 \%$ (normal $>52 \%$ ). There was a shift of center of gravity posteriorly, with less use of ankle strategy. Balance lab findings (Figure 2) showed definite affection of vestibulo spinal reflex, pointing to a likely pathology in the inner ear. Proprioception, vision and conflicting vision tests were normal. Patient was admitted in neurosurgery department and underwent discectomy in the last week of July 2001. After surgery the back pain reduced, but headache and vertigo persisted. She was advised isometric strengthening exercises, postural care, and weight reduction.

\section{Discussion}

MSM syndrome is an association of clinical features with radiological findings. The common clinical features are headache (migrainous), vertigo, hirsutism, menstrual disorders,

\section{Sensory Conditions Evaluation}

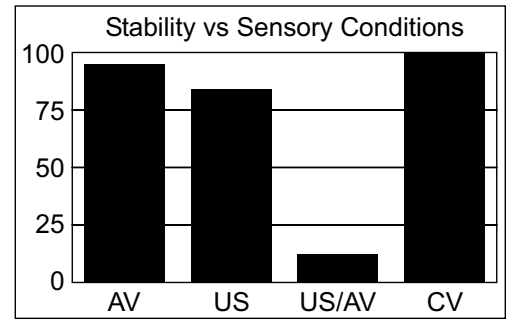

$\mathrm{AV}=$ Absent Vision $\quad$ US = Unstable Surface US/AV = Unstable Surface / Absent Vision $\mathrm{CV}=$ Conflicting Vision

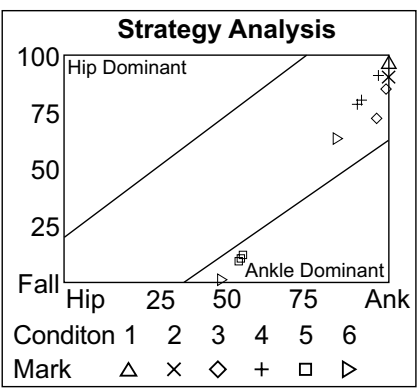

Data Range Note: NeuroCom Data Range: 20--59

Figure 2: Sensory organization test (SOT) report - decreased equilibrium score in absent vision and unstable surface galactorrhoea, obesity, depression, irritability, fatigability, transitory hemiplegias, hearing impairment, cranial nerve palsies, muscle weakness and seizures. ${ }^{[1-3]}$ The characteristic $\mathrm{X}$-ray finding in this disorder is thickening of inner table of skull. In 1936 Moore described it as Hyperostosis frontalis interna. However Ward observed these findings in older, emotionally disturbed females and in diabeties. ${ }^{[1]}$

The pathogenesis behind skull thickening is till now not clear. Moore reported it as a benign process that causes soft tissue compression with dural irritation and pressure atrophy of brain. ${ }^{[4]}$ Hasegawa et al and Latka et al described one case each with sclerotic skull bone causing compression on left frontal lobe and the headache in these patients resolved after surgery. ${ }^{[2,5]}$ There are reports with raised growth hormone levels, hyperparathyroidism, galactorrhoea, and hyperprolactinemia in these patients. ${ }^{[6,7]}$ Rosatti reported it as an autosomal dominant inheritance. ${ }^{[8]}$

The etiology for headache and vertigo is not clear but vascular cause is not ruled out. Our patient also had absent right radial pulse, to our knowledge this finding has not been reported previously. Recurrent cerebral circulatory disorders, sinus thrombosis, venous stasis on the optic disc with cerebral angiomas have also been reported..$^{[9,10]}$ The presence of deep sleep in our patient after an attack of vertigo may indicate vasovagal attack. Chrles $\mathrm{E}$ reported a case with narrowing of carotid arteries, in which vertigo and tinnitus improved after ventriculoatrial shunt. ${ }^{[1]}$ Harpman reported that vertigo was due to both central and peripheral origin. ${ }^{[12]}$

Balance is the ability to maintain the center of gravity over base of support. It has four components viz; vision, vestbular, proprioceptive sensation and biomechanical factors. Dynamic posturography works on these four mechanisms. There are six sensory organization tests. In test five eyes are closed and force plate are moved i. e. vision and proprioceptive sensation are eliminated. The patient has to maintain balance using only vestibular system; which is challenged by moving forceplates by the system automatically. In vestibular dysfunction, patients have more sway and equilibrium scores are also reduced with abnormal use of hip strategy. Our patient had nausea with fall and went unconscious for 30 minutes in this test with significant reduction $12 \%$ (normal-52\%) in equilibrium score. Composite score was also reduced $56 \%$ (normal$70 \%$ ). These test findings were also confirmed by vestibulometry findings.

In conclusion Morgagni-Stewart-Morel syndrome is one of the less understood and reported syndrome. The patient may present with varied symptoms and thus lead to difficulty in diagnosis. A thorough clinical examination with neurological finding can help to diagnose this rare entity. In addition to other reported clinical features, we found absent redial pulse and impaired balance due to vestibular dysfunction. Dynamic posturography is one of the tests that help to assess vestibular dysfunction more objectively. 


\section{References}

1. Capraro VJ, Dillon WP, Calabrese JS. Morgagni's Syndrome Metabolic Craniopathy. Obst and Gyne 1970;35:565-9.

2. Hasegawa T, Ito H, Yamamoto S, et al. Unilateral hyperostsis frontalis interna J Neurosurg 1983;59:710-3

3. Brij K, Singh MM, Seth HC, et al. Morgagni Syndrome A case report. J Ind Med Assoc 1972;58:376-8.

4. Moore S. Metabolic craniopathy. AJR 1936;35:30-9.

5. Latka D, Szydlik W, Glaubic-Latka, et al. A Case of Morgagni -Morel-Stewart syndrome with violent headaches predominant in the clinical course treated surgically. Neurol Neurochir Pol 1995;29:253-6.

6. Koev D, Milanov S, Koeva L, et al. Plasma growth hormone level in the Morgagni-Stewart-Morel syndrome. Endocrinologie 1978;16:65-8.

7. Pawlikowski M, Komorowski J. Hyperostosis frontalis, galactorrhoea / hyperprolactinaemia and Morgagni-Stewart-Morel syndrome. Lancet $1983 ; 26 ; 1: 474$

8. Rosatti. Family affected by hyperostosis frontalis interna (Morgagni-Morel Syndrome) through 4 successive generations. J Genet Hum 1972;20:207-52.

9. Radek A, Piwowarski W, Maciejezak A. Unilateral hyperostosis frontalis interna coexistent with cerebral angioma. Neurolo Neurochir Pol 1987;21:261-4.

10. Pribylova NN, Shkliarova BS, Fomin AV. Morgagni-Stewart-Morel syndrome with recurrent cerebral circulatory disorders. Klin Med (Mosk) $1987 ; 65: 142-3$

11. Charles E. Morgagni syndrome and Hyperostosis Frontalis interna. Lancet 1974;30:1331-2.

12. Harpman JA. Vestibulometry in a case of Morgagni-Stewart-Morel Syndrome. J Laryngol Otol 1972;86:63-6.

Accepted on 10.11.2002 